Article

\title{
Investigation of C-K Theory Based Approach for Innovative Solutions in Bioinspired Design
}

\author{
Prabaharan Graceraj P. ${ }^{1}$, Jacquelyn K. Nagel ${ }^{2}$, Christopher S. Rose ${ }^{3}$ and Ramana M. Pidaparti ${ }^{1, *}$ \\ 1 School of Environmental, Civil, Agricultural and Mechanical Engineering, University of Georgia Athens, \\ Athens, GA 30602, USA \\ 2 College of Engineering, James Madison University, Harrisonburg, VA 22807, USA \\ 3 Biology Department, James Madison University, Harrisonburg, VA 22807, USA \\ * Correspondence: rmparti@uga.edu; Tel.: +1-706-542-4057
}

Received: 28 June 2019; Accepted: 18 July 2019; Published: 21 July 2019

check for updates

\begin{abstract}
This paper discusses the investigation of a Concept-Knowledge (C-K) theory based approach for generating innovative design solutions in bioinspired design projects. Undergraduate students enrolled in sophomore engineering design courses at both the University of Georgia (UGA) and James Madison University (JMU) completed bioinspired design projects using C-K theory based templates. Hypothesis testing, principal component analysis (PCA) and support vector machine (SVM) techniques were applied on the students' performance scores of a C-K theory based bioinspired design process to identify the biomimicry attributes which supported the evolution of innovative design solutions. Results from the analysis suggest that the C-K theory based approach is useful for generating innovative design solutions.
\end{abstract}

Keywords: biomimicry; innovative design; interdisciplinary; quantitative analysis

\section{Introduction}

Design engineering research communities are paying increased attention to developing new strategies that result in more creative and innovative design solutions [1]. Several empirical and analytical studies have modeled key aspects involved in developing creative and innovative solution spaces in the engineering design process. Some of the research investigations revealed that developing a relationship framework model that connects the design problem, scientific knowledge and solution space by analysis, synthesis and evaluation activities would help to refine and structure the design process to obtain creative and innovative design solutions [2-5]. Further, new and emerging methodological frameworks such as bioinspired design processes were also identified as potential techniques for generating innovative design solutions from biological systems to address various engineering design problems [6,7].

Several unique characteristics of bioinspired design process which stimulate innovation and creativity in design solutions have been identified including interdisciplinary subject-matter, representation of themes, objects and relations with different terminology, expression of design problems and solutions in different perspectives, complex and multifunctional biological design aspects and contrast in resource domains [8]. In spite of its many interesting characteristics, the bioinspired design process is still lagging in systemizing the analogical retrieval of biological knowledge and transferring the same into design solutions [9]. Hence, investigations focusing on systematic approaches in bioinspired design are increasing in number to address the issues related to the development of bioinspired design engineering models. These models focus on obtaining innovative design solutions for engineering design problems by mapping the design process through the collection, integration and analysis of biological knowledge and information using various tools and techniques [10-12]. 
Computational and framework-based approaches have been used in the bioinspired design process literature, to develop design process models. In a computational based approach, a function-based model was employed to obtain innovative concepts or design solutions with the integration of engineering and biology thesauruses with organized search tools $[13,14]$. In a framework-based modeling technique, the relationship between biomimetic and management theory was explored and the impact of market orientation, entrepreneurial orientation, technology orientation, learning orientation and networking orientation was analyzed to design innovative products and services [15]. These researchers investigated the concept generation process in the context of ideas, analogy, blending and so on and emphasized the importance of the knowledge structure in the concept generation process. Design engineering researchers also attempted to uncover the logic of the creative process in design engineering with a structure of two spaces and developed a new theory called Concept-Knowledge (C-K) theory [16,17]. Further, to illustrate and to map the roles of biological and scientific knowledge in the bioinspired design processes, a C-K theory based bioinspired design process model has also been proposed [18-20]. This model was represented as a series of steps involving activation of biological knowledge, expansion of biological and traditional knowledge, exploration of the traditional knowledge and concepts, and portioning and integration of traditional knowledge space to define a solution path. Attempts were made to explore the $\mathrm{C}-\mathrm{K}$ theory based bioinspired design process model through the teaching - learning process in undergraduate bioinspired design engineering courses using qualitative analysis techniques. It was found that the C-K theory based bioinspired design process resulted in significant student learning and engagement [21-23].

In the context of addressing the research gap in demonstrating the capability of the C-K theory based bioinspired design process to produce innovative design solutions and to define design paths more elaborately with statistical and deep learning techniques, we developed a linear combination model with biomimicry and design engineering attributes and tested the following hypotheses:

Hypothesis 1. The C-K theory based bioinspired design process model has significant transferability for use across different institutions.

Hypothesis 2. The C-K theory based bioinspired design process model has significant capability to produce innovative design solutions.

Hypothesis 3. The C-K theory based bioinspired design process model has significant capability to define a design process.

Hypothesis 1 was framed to demonstrate the proposed model's capability in obtaining innovative solutions from the users of different institutions and universities. Hypotheses 2 and 3 were formulated to demonstrate and to describe the model's capability to produce innovative solutions and also to define design paths.

\section{Methods}

\subsection{C-K Theory Based Bioinspired Design Process}

The design process model used in our research investigation is based on the C-K theory approach for innovative design solutions which modeled the dynamics of the design process as a joint-expansion of space objects [16]. In our model, the design concepts and solutions were developed by the reasoning and logical thoughts provoked by biological and traditional knowledge. The entire model was constructed with two major distinguishable spaces namely concept $(C)$ and knowledge space (K) and with their expanded sub spaces. The subspaces in $\mathrm{C} \& \mathrm{~K}$ space would initiate the process of inquiring into the biological system, obtaining design concepts by mimicking biological systems, controlling the evolution of design solution from problem definition and helping to define the design path. The subspace designated as C0 in C-space would help to define the design problem and would 
act as a place of origination to start the design solution evolution process. The other C-subspace $\mathrm{C} 1$ would act as a bridging space with details for the development of design concepts in concept subspace $\mathrm{C} 2$ by inheriting its properties from the traditional and biological knowledge space objects. The subspace labeled C3 would represent the bioinspired design solution as a result of interaction of properties of all the objects in $\mathrm{C}$ and $\mathrm{K}$ spaces. The properties of association and relations enabled information flow between subspaces that inherited their properties from design and knowledge space objects. Similarly, the knowledge space of our model was constructed with traditional and biological knowledge subspaces. The traditional knowledge subspace in knowledge space derived its properties from similar or the same class of design solutions, or the scientific methods used for solving design problems. The biological knowledge subspace was defined with the unique property that encapsulates the required biological information to exhibit innovative design solutions. In the entire mapping process of concepts and knowledge, the integration and association of subspaces was portrayed by four operations, namely, expansion of knowledge space, expansion of the concept space, concept generation from knowledge, and knowledge generation from concepts. Four C-K operators were used to describe these operations. The design concept evolution of bioinspired design process from concept subspace is described with $\mathrm{C} \rightarrow \mathrm{C}$ operators. The knowledge resulting from knowledge spaces is represented by $\mathrm{K} \rightarrow \mathrm{K}$ operators while the relationship between knowledge spaces and concept spaces is mapped by $\mathrm{K} \rightarrow \mathrm{C}$ and $\mathrm{C} \rightarrow \mathrm{K}$ operators [24]. The evolution of a bioinspired design solution in $\mathrm{C}-\mathrm{K}$ space as described above is illustrated with an example case in Figure 1 for the design task of developing a mechanism for a self-propulsion system. In order to investigate the capabilities of the C-K theory based bioinspired design process to produce innovative solutions, we conducted statistical analyses on student performance scores on this task as discussed below.

Concept

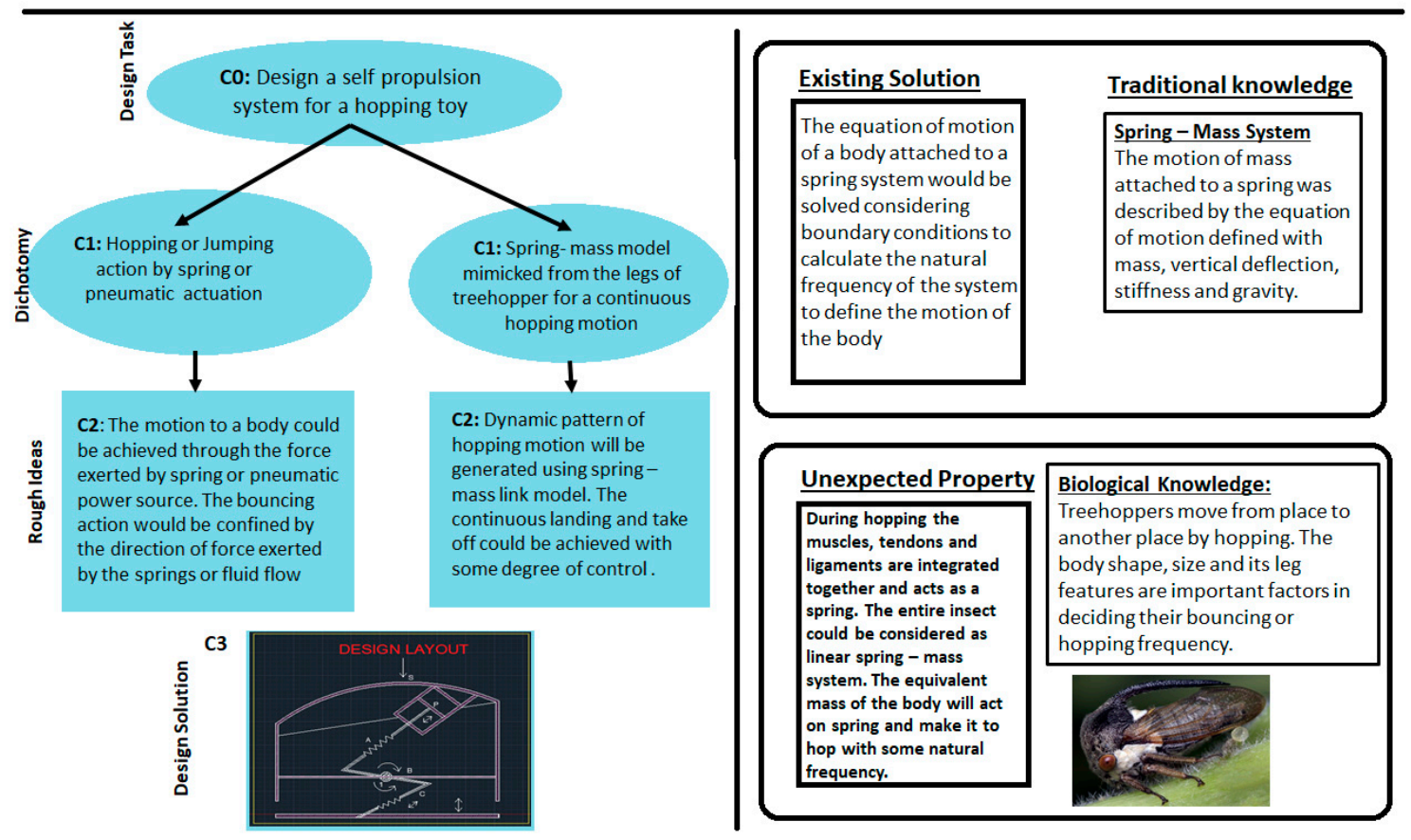

Figure 1. Example of bioinspired design solution in Concept-Knowledge space.

\subsection{Motivational Data for Statistical Analysis}

A bioinspired design course that familiarizes engineering undergraduate students with bio-inspiration concepts to solve design problems was introduced to sophomore engineering students at James Madison University (JMU) and the University of Georgia (UGA). As a part of their course work, the students were asked to map the bioinspired design process of a self-propulsion system using 
the $\mathrm{C}-\mathrm{K}$ theory-based bioinspired design process model described in the previous section. The number of students, who completed this project, were $n_{j}=53$ at JMU and $n_{u}=48$ at UGA. We decided to evaluate all the student performance on this design project work following an evaluation protocol. Based on student's performance on biomimicry and design engineering attributes, score values on an ordinal scale were assigned to each attribute and a data matrix for the analysis was developed to carryout statistical analyses.

\subsection{Evaluation Protocol for Design Performance}

As the main aim of our study was to examine the role of C-K theory based bioinspired design process in producing innovative design solutions and to understand its characteristics, the analysis of student performance was carried out on two major aspects: biomimicry process aspects and engineering design aspects. Further, for the effective analysis of student performance, various biomimicry and design engineering process attributes were defined as part of a performance measurement protocol that would conceptualize the design as a framework with attributes which would elaborate bioinspired design solution evolution aspects.

The biological and natural systems have enormous information and knowledge for solving engineering and scientific problems and bioinspired design process is the gateway for obtaining innovative solutions from them. The power of the bioinspired design process lies in its ability to extract information or knowledge at different levels of solution evolution from biological knowledge resources [25]. In addition to the above biological information and knowledge handling or analysis aspects of the bioinspired design process, we identified other elements such as understanding analogies in two different systems, defining biological knowledge as a group of available solutions, and defining design concepts and design processes as relational objects that could be used to characterize the bioinspired design process and decided to consider them in defining biomimicry attributes for our research study. Similarly, we examined various stages of the engineering design process as outlined in research based pedagogical practices to finalize our design process attributes [26,27]. In addition to that, best practice in the design solution evolution of new product design and existing product development for sustainability in the industrial scenario were also analyzed to define the design engineering attributes. During a new product design, the designer's imagination will initiate the action of accessing the scientific and engineering knowledge resources at the various stages of innovative solution development. The design process will logically progress towards solution from one stage to another stage by a sequence of decision making actions. The progress of the design process in terms of generating innovative design solution would effectively be controlled by the feasibility analysis and by many motivational factors. The same phenomenal aspects could also be applied to product development processes, except that the scientific or engineering information about the existing product would dominate in initiating the design solutions. In the engineering design process, the innovative design concepts are the outcome of the designer's structured thoughts or the imagination process triggered by knowledge or information resources. The innovative ideas provoked in the thought process would be subjected to a continuous pattern of changes to result in design solutions. In order to characterize student projects in different stages of design solution development across two different aspects, we developed biomimicry and design engineering attributes, as described above and presented in Table 1.

After determining biomimicry and design engineering attributes for the analysis, we assigned ordinal score values of 1, 2, 3 and 4 for the students' capability to effectively translate the biological knowledge into design solutions. These four score values were used to assign four categorical performance ratings of students on each attribute. The criteria for assignment of score values for each attribute is presented in Table 2. 
Table 1. Biomimicry and Design Engineering Attributes for Statistical Analysis.

\begin{tabular}{cc}
\hline Biomimicry Attributes (BM) & Design Engineering Attributes (DE) \\
\hline Relating Biological Property (BM1) & Imagination (DE1) \\
Biological knowledge (BM2) & Innovation (DE2) \\
Traditional knowledge (BM3) & Problem definition (DE3) \\
Defined Rough idea (BM4) & Defining Solution Methods (DE4) \\
Transition from rough idea to sketch (BM5) & Decision making (DE5) \\
Definition of design path (BM6) & Implementation and improvement (DE6) \\
Sketches/design output (BM7) & Active participation (DE7) \\
\hline
\end{tabular}

Table 2. Criteria for Assignment of Score Value to Each Attribute.

\begin{tabular}{cc}
\hline Score Value & Criteria \\
\hline 4 & $\begin{array}{c}\text { For a feasible and innovative design solution generated by relating } \\
\text { biological knowledge, with a clear understanding of biological and } \\
\text { traditional knowledge, and well-defined/highly creative concepts and } \\
\text { ideas with a well-defined design path which was evolved due to } \\
\text { logically connected decisions and with well-mapped traditional and } \\
\text { biological knowledge that led to creative solution of design problems } \\
\text { For feasible design solutions with few existing design features, some } \\
\text { missing biological property relations, moderate explanation of biological } \\
\text { and traditional knowledge, design concepts with a moderately defined } \\
\text { design path and with moderately linked traditional and biological } \\
\text { knowledge for solution of design problems } \\
\text { For the design solution with suggested improvements, fewer or missing } \\
\text { biological property relations, lower level explanation of biological and } \\
\text { traditional knowledge, design concepts with a roughly defined design } \\
\text { path and with roughly linked traditional and biological knowledge for } \\
\text { solving design problems } \\
\text { For design solutions with least feasibility, poorly related biological } \\
\text { properites, poor explanation of biological and traditional knowledge, } \\
\text { design concepts with an unclear design path and with poorly linked } \\
\text { traditional and biological knowledge for solution of design problems }\end{array}$ \\
\end{tabular}

\subsection{Application of Performance Measurement Protocol}

The performance measurement protocol as defined above was applied on JMU and UGA students' project work and the quality of bioinspired design thinking as well as their ability to translate this design thinking into a design solution was analyzed, as illustrated by the example case presented in Figure 2.

The example student project case illustrated above involves bioinspired design concept development for a propulsion system that decreases the effort applied by the bicycle rider. This design concept was inspired by the example of an ant's legs that are strong and flexible and act as springs that enable locomotion. It is clear from this project presentation that the C-K theory based bioinspired design process played a major role in the designer's thinking process and culminated in an innovative design solution. The student began her work with a clear problem definition and started her search in the knowledge space for initial concept development. The knowledge space in the C-K map was helpful in organizing biological knowledge and also for the effective retrieval of knowledge from the space. The unexpected property extraction from biological knowledge and application of this knowledge in the engineering space resulted in an innovative design. The unexpected property acted as a bridging object that connected biological and scientific or engineering knowledge. The details of concepts learned from the biological system are detailed in the concept space. The existing propelling systems and working principles for higher propelling power are explained in the knowledge space of the C-K map and this information is helpful for the translation of ideas into design concepts. The 
propelling system inspired by ant legs is detailed and its properties are key to the bioinspired design solution. The design concept is connected with knowledge space objects in the sketching through C3 object definitions.

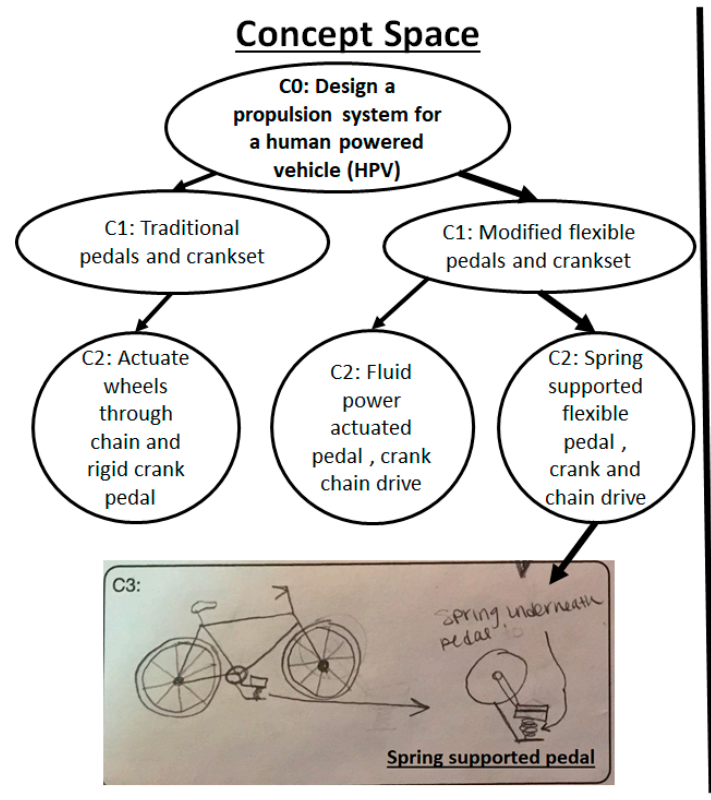

Knowledge Space

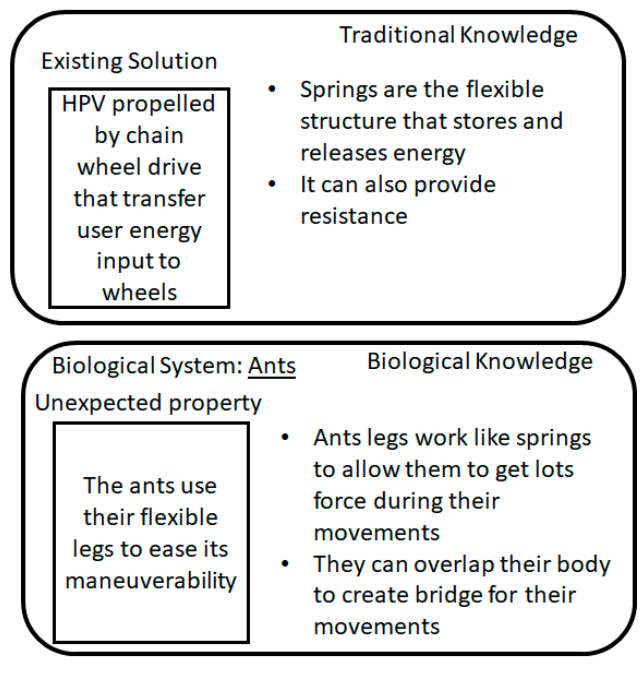

Figure 2. Evaluation of C-K theory based design process.

All the C-K theory-based design solutions of the students at UGA and JMU were analyzed as illustrated in the above example and performance scores on each attribute for each student project were assigned and the performance score matrix was completed for statistical analysis.

\subsection{Statistical Analysis}

Statistical analysis methods such as test of hypothesis, principal component analysis (PCA) and support vector machine (SVM) classifier were among the tools used in our research study to analyze student performance and to elaborate the key features of the bioinspired design process that led to innovative design solutions.

As the performance score data considered for our analysis was not based on any standard distributions, a non-parametric statistical method, the two sample Kolmogorov-Smirnov test was used to test the first hypothesis defined in Section 1 (the two sample data sets from JMU and UGA and their attributes are from the same population) [28,29]. The statistical data models with biomimicry and design engineering attributes J_BM1, J_BM2, . . . .J_BM7, J_DE1, J_DE2 . . . J_DE7 and U_BM1, U_BM2, .....U_BM7, U_DE1, U_DE2 ... .U_DE7 of JMU and UGA datasets were developed and passed as arguments in the Matlab function to test Hypothesis 1.

The datasets considered for our investigation were defined with multiple attributes. Therefore, data mining tools such as PCA and SVM classifier were used to elucidate the underlying knowledge in the datasets [30]. Initially, to select the most relevant design engineering attributes that represented all the design engineering attributes, observations in design engineering attributes were transformed orthogonally towards the direction of large variance by PCA. For conducting PCA on design engineering attributes observations, the performance score data matrix denoted by $\mathrm{Z}$ with the dimensional attributes of (DE1, DE2, ... , DE7) for the pooled JMU and UGA data was created. Then, the variance-covariance matrix of the dataset $(Z)$, denoted by $\sum$ was calculated and was solved by using the Matlab function to find principal components coefficients, principal component scores for each attribute and eigenvalues of variance-covariance matrices. The cumulative variance explained by the number of principal components was calculated and the scree plot was plotted to decide the number of principal components 
for our study [31]. After deciding the number of principal components, the biplot was plotted to study the correlation between attributes from the magnitude and the direction of vector plots corresponding to each attribute [32-34].

Furthermore, to find the biomimicry attributes which played a major role and resulted in innovative design solutions, the datasets were trained using a supervised deep learning approach, and a support vector machine (SVM) classifier, a binary classification algorithm (which has been applied in different fields such as character segregation, image analysis, etc.) was used for the effective classification and clustering of observations to study the underlying characteristics of the data $[35,36]$. In our study, we considered all seven biomimicry attributes as predictor variables and developed response variables with two label values ' 1 ' and ' 0 ' based on the performance score values in innovation and imagination of the design engineering attributes, reduced by the PCA method. The observations labeled as class ' 1 ' were creative and innovative design solutions, and the observations labeled as class ' 0 ' were not creative and innovative design solutions. The median score values of DE1 and DE2 were chosen as a limit value to classify the observations in predictor variables to either 'class 1 ' or 'class 0 '. After determining values of response variables for JMU and UGA datasets from design engineering attributes DE1 and DE2, the datasets were trained using linear, polynomial and Gaussian SVM classifier in Matlab. It was observed during classification analysis that the linear SVM model was more accurate (87\% classification accuracy, plot $\mathrm{H}$ in Figure 5) compared to the polynomial and Gaussian SVM models. Hence, the linear SVM was chosen to train pooled JMU and UGA dataset with selected biomimicry attributes-BM1, through BM7.

\section{Results and Discussion}

\subsection{Two Sample Kolmogorov-Smirnov Test}

The Matlab function returned two sample Kolmogorov-Smirnov test results in ' $h$ ' and ' $p$ ' vectors. It was found from the output that the null hypothesis decision values in vector ' $h$ ' were zero for all attribute pairs. This showed that the null hypothesis-the performance score data of attributes pairs from JMU and UGA datasets were from the same population. The statistical inference obtained from this finding is that the institutional dependence criteria of C-K theory based bioinspired design process does not influence its performance. In other words, both JMU and UGA datasets were appropriate to use as a pooled single dataset for the prediction of relationship between biomimicry and design engineering attributes using PCA and SVM methods.

\subsection{On Design Engineering Attributes Dimensions Reduction by PCA}

Initially, the cumulative variance explained by principal components was calculated and plotted in a scree plot, as shown in Figure 3 for the pooled JMU and UGA dataset. This value was used to choose the number of principal components. From the Pareto charts of the pooled JMU and UGA data set, it was found that $67 \%$ of the variance was explained by the first two principal components. Hence, the principal component's scores of the first two principal components were used to identify the best representative design engineering attributes of the students' design performance scores [31]. Secondly, the principal components coefficients and principal components scores of each design engineering attribute of pooled JMU and UGA data were plotted as vectors and scatter points in a biplot with reference to the two principal axes as shown in Figure 4.

The biplot of pooled JMU and UGA datasets showed the relative importance of design engineering attributes in principal score space by the direction and magnitude of attribute vectors. It was observed from the biplots that the design engineering attributes DE1 (Innovation) and DE2 (Imagination) had the highest magnitude in the positive quadrant for the pooled dataset. Hence, the design engineering attributes DE1 and DE2 were chosen as the best attributes to represent the design performance data with a $67 \%$ variability threshold limit and also as response variables for the classification study using the SVM method. 


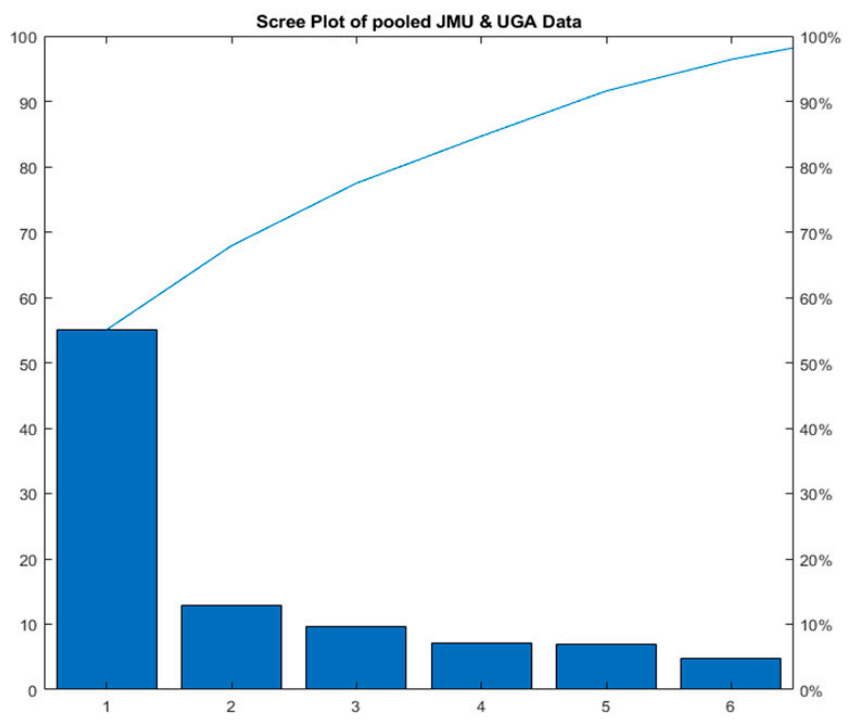

Figure 3. Scree plot of pooled JMU and UGA datasets to decide number of principal components.

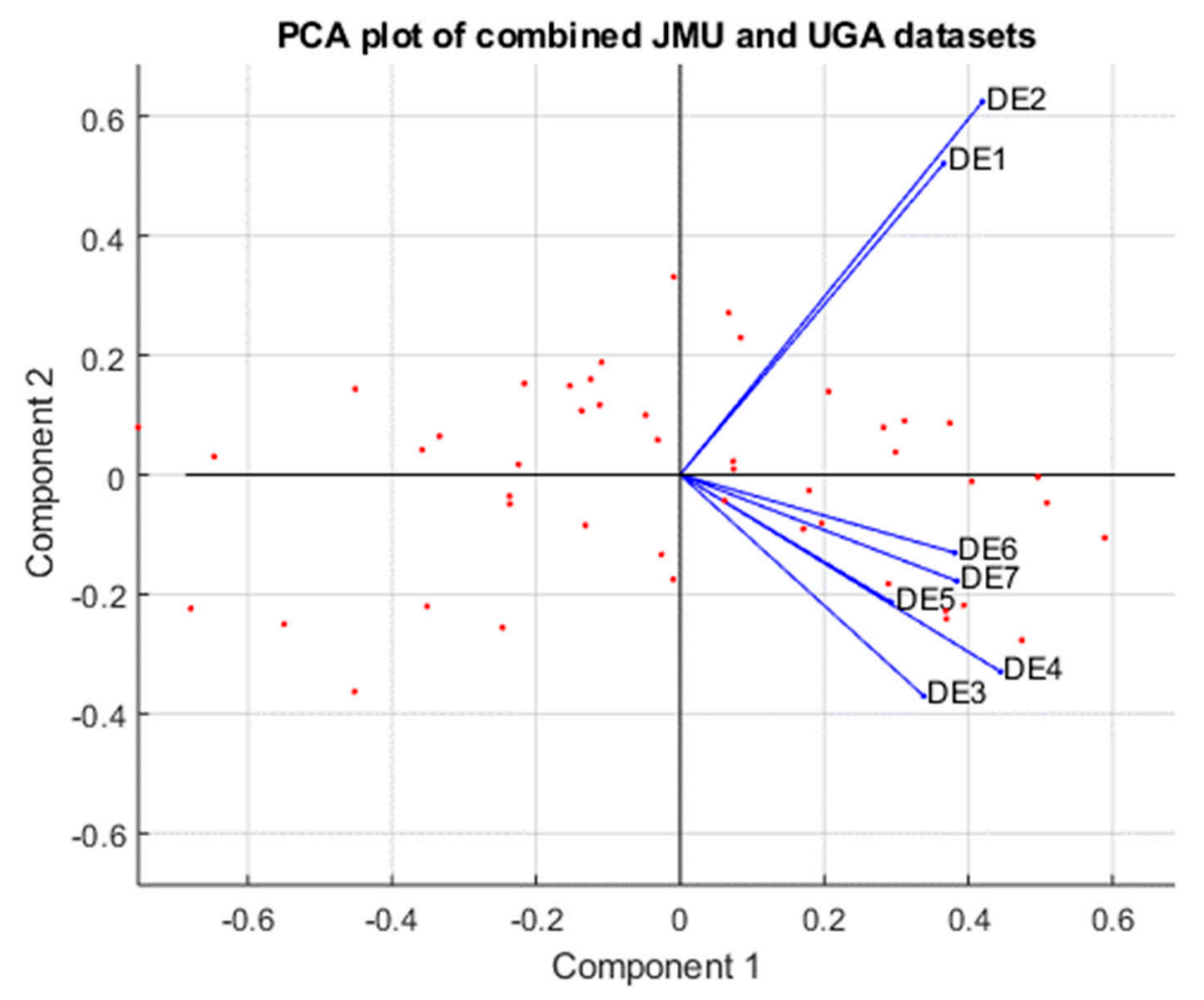

Figure 4. PCA biplot of pooled JMU and UGA dataset for design engineering attributes.

\subsection{SVM Classification Analysis}

The data model developed from pooled JMU and UGA performance scores as trained with 10 fold cross validation by a linear SVM algorithm. The receiver operating characteristic (ROC) curves for the trained model with selected attributes were plotted as presented in Figure 5 (Plot A to G for selected attributes and Plot $\mathrm{H}$ with all attributes), to understand the role of each biomimicry attribute (predictor variable) in achieving innovative design solutions (response variable). As the SVM is the deep learning technique which analyzes false correlations of attributes through classification accuracy, we used area under curve (AUC) of ROC as a measure of classification performance of our supervised deep learning method on bioinspired design performance score data [37,38]. 

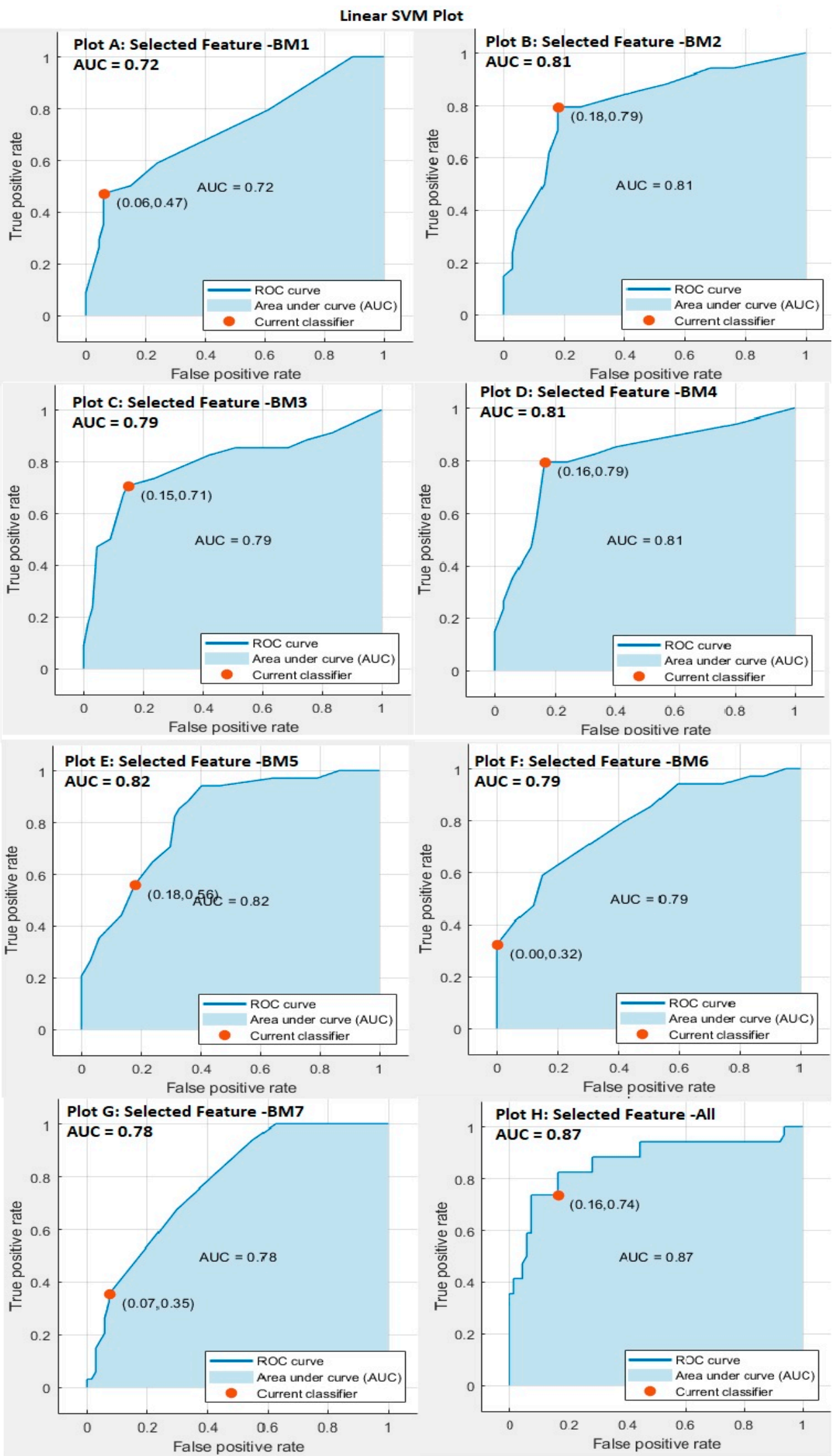

Figure 5. The ROC curves of pooled JMU and UGA dataset with selected biomimicry attributes-Plot A to $\mathrm{G}$ and all biomimicry attributes-Plot $\mathrm{H}$. 
The AUC values of 0.72 to 0.82 (Plot A to G) were observed in the SVM classification analysis with selected biomimicry features (i.e., predictor variables) of biomimicry attributes 1 to 7 (BM1, BM2 ... \& BM7) according to the binary design innovation ability scale used in our study. This classification analysis indicated that the performance score data set of each biomimicry attribute effectively contributed to the response dataset vector of the design innovation binary classification attribute. A higher percentage of observations in biomimicry attributes were effectively classified as producing innovative design solutions and a lower percentage of observations were clustered in another class that did not produce innovative design solutions. Analyses on observations of clusters that produced innovative design solutions in the context of the C-K theory based approach were completed to overcome design fixation effects to get innovative design solutions [39-42]. It was evident from the analysis of student performance on each attribute that the students were able to obtain necessary knowledge, search for alternatives and experience collaborative creative thinking that resulted in innovative design solutions. It can also be concluded based on the classification of student performance on each attribute of the bioinspired design process, mapped in C-K space, that the knowledge generated and accessed by the design process for the evolution of innovative design solution from the concepts and problem definitions was effectively controlled by students' design thinking. The relational mapping process enhanced the design process evolution and to the generation of innovative design solutions.

\section{Conclusions}

In this study, the bioinspired design process mapped in C-K space was analyzed using statistical and deep learning methods to find its capability to produce innovative design solutions. The JMU and UGA students' design performance score datasets were considered for the analysis. The Kolmogorov-Smirnov test showed that the C-K theory based bioinspired design process was not institutionally dependent and would exhibit same performance in all institutes. The PCA and SVM analysis of the data revealed that the C-K theory based bioinspired design process had significant capability to produce innovative design solutions and could define a design path or process to obtain innovative design solutions. Future research will focus on a prototype design development process of a bioinspired product to evaluate the performance of the C-K theory-based bioinspired design process model.

Author Contributions: Conceptualization-R.M.P. and P.G.P.; Methodology, Analysis, Investigation \& Writing manuscript-P.G.P.; Data contribution, Manuscript review-R.M.P., J.K.N. and C.S.R.; Supervision-R.M.P.

Funding: This work was supported by the National Science Foundation under grant number \# NSF-1504614.

Acknowledgments: The authors would like to thank JMU and UGA engineering students for their participation in this study. Any opinions, findings and conclusions or recommendations expressed in this material are those of the authors and do not necessarily reflect the views of the National Science Foundation.

Conflicts of Interest: The authors declare no conflict of interest.

\section{References}

1. Andriopoulos, C.; Lewis, M.W. Managing innovation paradoxes: Ambidexterity lessons from leading product design companies. Long Range Plan. 2010, 43, 104-122. [CrossRef]

2. Cross, N. Descriptive models of creative design: Application to an example. Des. Stud. 1997, 18, 427-440. [CrossRef]

3. Gero, J.S.; Mc Neill, T. An approach to the analysis of design protocols. Des. Stud. 1998, 19, 21-61. [CrossRef]

4. Dorst, K.; Cross, N. Creativity in the design process: Co-evolution of problem-Solution. Des. Stud. 2001, 22, 425-437. [CrossRef]

5. Schønheyder, J.F.; Nordby, K. The use and evolution of design methods in professional design practice. Des. Stud. 2018, 58, 36-62. [CrossRef]

6. Bar-Cohen, Y. Biomimetics-Using nature to inspire human innovation. Bioinspiration Biomim. 2006, 1, 1-12. [CrossRef] [PubMed] 
7. Moreno, D.P.; Hernández, A.A.; Yang, M.C.; Otto, K.N.; Hölttä-Otto, K.; Linsey, J.S.; Wood, K.L.; Linden, A. Fundamental studies in design-by-analogy: A focus on domain-knowledge experts and applications to transactional design problems. Des. Stud. 2014, 35, 232-272. [CrossRef]

8. Helms, M.; Vattam, S.S.; Goel, A.K. Biologically inspired design: Process and products. Des. Stud. 2009, 30, 606-622. [CrossRef]

9. Nagel, J.K. Systematic bio-inspired design: How far along are we? Insight 2016, 19, 32-35. [CrossRef]

10. Nagel, J.K.; Stone, R.B. A systematic approach to biologically-inspired engineering design. In Proceedings of the ASME 2011 International Design Engineering Technical Conferences and Computers and Information in Engineering Conference, Washington, DC, USA, 28-31 August 2011; pp. 153-164.

11. Vandevenne, D.; Verhaegen, P.A.; Dewulf, S.; Duflou, J.R. Product and organism aspects for scalable systematic biologically-inspired design. Proc. Eng. 2015, 131, 784-791. [CrossRef]

12. Kennedy, E.; Niewiarowski, P. Biomimicry: Do frames of inquiry support search and identification of biological models? Designs 2018, 2, 27. [CrossRef]

13. Nagel, J.K.; Stone, R.B.; McAdams, D. An engineering-to-biology thesaurus for engineering design. In Proceedings of the ASME 2010 International Design Engineering Technical Conferences and Computers and Information in Engineering Conference, Montreal, QC, Canada, 15-18 August 2010.

14. Nagel, J.K.; Stone, R.B.; McAdams, D.A. Function-based biologically inspired design. In Biologically Inspired Design: Computational Methods and Tools; Goel, A.K., McAdams, D.A., Stone, R.B., Eds.; Springer: London, UK, 2014; pp. 95-125.

15. Ulhøi, J.P. Framing biomimetics in a strategic orientation perspective (biopreneuring). Technol. Anal. Strateg. Manag. 2015, 27, 300-313. [CrossRef]

16. Hatchuel, A.; Weil, B. A new approach of innovative design: An introduction to CK theory. In Proceedings of the ICED 03, the 14th International Conference on Engineering Design, Stockholm, Sweden, 19-21 August 2003; pp. 109-110.

17. Hatchuel, A.; Weil, B. C-K design theory: An advanced formulation. Res. Eng. Des. 2009, 19, $181-192$. [CrossRef]

18. Hatchuel, A.; Le Masson, P.; Weil, B. The design of science based products: An interpretation and modelling with C-K theory. In Proceedings of the 9th International Design Conference, DESIGN 2006, Dubrovnik, Croatia, 15-18 May 2006; pp. 33-44.

19. Salgueiredo, C.F.; Hatchuel, A. Modeling biologically inspired design with the C-K design theory. In Proceedings of the DESIGN 2014 13th International Design Conference, Dubrovnik, Croatia, 19-22 May 2014; pp. 23-32.

20. Salgueiredo, C.F.; Hatchuel, A. Beyond analogy: A model of bioinspiration for creative design. Artif. Intell. Eng. Des. Anal. Manuf. 2016, 30, 159-170. [CrossRef]

21. Nagel, J.K.; Stone, R.B. Teaching biomimicry in the context of engineering design. In Proceedings of the Biomimicry in Higher Education Webinar, Missoula, MT, USA, 29 January 2011; pp. 61-68.

22. Nagel, J.K.; Pidaparti, R.M. Significance, prevalence and implications for bio-inspired design courses in the undergraduate engineering curriculum. In Proceedings of the ASME 2016 International Design Engineering Technical Conferences and Computers and Information in Engineering Conference, Charlotte, NC, USA, 21-24 August 2016.

23. Nagel, J.; Pittman, P.; Pidaparti, R.; Rose, C.; Beverly, C. Teaching bioinspired design using C-K theory. Bioinspired Biomim. Nanobiomater. 2017, 6, 1-10. [CrossRef]

24. Hatchuel, A.; Le Masson, P.; Weil, B. C-K theory: Modelling creative thinking and its impact on research. In Creativity, Design Thinking and Interdisciplinarity; Springer: Berlin, Germany, 2017; pp. 169-183.

25. Weidner, B.V.; Nagel, J.; Weber, H.-J. Facilitation method for the translation of biological systems to technical design solutions. Int. J. Des. Creat. Innov. 2018, 6, 211-234. [CrossRef]

26. Safoutin, M.J.; Atman, C.J.; Adams, R.; Rutar, T.; Kramlich, J.C.; Fridley, J.L. A design attribute framework for course planning and learning assessment. IEEE Trans. Educ. 2000, 43, 188-199. [CrossRef]

27. Woollacott, L.C. Validating the CDIO syllabus for engineering education using the taxonomy of engineering competencies. Eur. J. Eng. Educ. 2009, 34, 545-559. [CrossRef]

28. Hildebrand, D.K. Analysis of Ordinal Data; Sage Publications: Beverly Hills, CA, USA, 1977.

29. Corder, G.W. Nonparametric Statistics for Non-Statisticians a Step-By-Step Approach; Wiley: Hoboken, NJ, USA, 2009. 
30. Granato, D.; Santos, J.S.; Escher, G.B.; Ferreira, B.L.; Maggio, R.M. Use of principal component analysis (PCA) and hierarchical cluster analysis (HCA) for multivariate association between bioactive compounds and functional properties in foods: A critical perspective. Trends Food Sci. Technol. 2018, 72, 83-90. [CrossRef]

31. Zhu, M.; Ghodsi, A. Automatic dimensionality selection from the scree plot via the use of profile likelihood. Comput. Stat. Data Anal. 2006, 51, 918-930. [CrossRef]

32. Abdi, H.; Williams, L.J. Principal component analysis. Wiley Interdiscip. Rev. Comput. Stat. 2010, 2, 433-459. [CrossRef]

33. Aouabdi, S.; Taibi, M.; Bouras, S.; Boutasseta, N. Using multi-scale entropy and principal component analysis to monitor gears degradation via the motor current signature analysis. Mech. Syst. Signal Process. 2017, 90, 298-316. [CrossRef]

34. Nobi, A.; Alam, S.; Lee, J.W. Dynamic of consumer groups and response of commodity markets by principal component analysis. Phys. A Stat. Mech. Its Appl. 2017, 482, 337-344. [CrossRef]

35. Burges, C.J.C. A tutorial on support vector machines for pattern recognition. Data Min. Knowl. Discov. 1998, 2, 121-167. [CrossRef]

36. Ling, C.X.; Huang, J.; Zhang, H. AUC: A better measure than accuracy in comparing learning algorithms. In Proceedings of the 16th Conference of the Canadian Society for Computational Studies of Intelligence, AI 2003, Halifax, NS, Canada, 11-13 June 2003; pp. 329-341.

37. Joachims, T. Text categorization with support vector machines: learning with many relevant features. In Proceedings of the 10th European Conference on Machine Learning, Chemnitz, Germany, 21-23 April 1998; pp. 137-142.

38. Lin, S.-W.; Lee, Z.-J.; Chen, S.-C.; Tseng, T.-Y. Parameter determination of support vector machine and feature selection using simulated annealing approach. Appl. Soft Comput. 2008, 8, 1505-1512. [CrossRef]

39. Hatchuel, A.; Le Masson, P.; Weil, B. Learning to Face the Unknown and the Emergent: A Project-Based Critical Learning Perspective; European Academy of Management: Ljublana, Slovenia, 2008; p. 19.

40. Hatchuel, A.; Le Masson, P.; Weil, B. Teaching innovative design reasoning: How concept-knowledge theory can help overcome fixation effects. Artif. Intell. Eng. Des. Anal. Manuf. 2011, 25, 77-92. [CrossRef]

41. Agogué, M.; Le Masson, P.; Dalmasso, C.; Houdé, O.; Cassotti, M. Resisting classical solutions: The creative mind of industrial designers and engineers. Psychol. Aesthet. Creat. Arts 2015, 9, 313-318. [CrossRef]

42. Agogué, M.; Poirel, N.; Pineau, A.; Houdé, O.; Cassotti, M. The impact of age and training on creativity: A design-theory approach to study fixation effects. Think. Ski. Creat. 2014, 11, 33-41. [CrossRef]

(C) 2019 by the authors. Licensee MDPI, Basel, Switzerland. This article is an open access article distributed under the terms and conditions of the Creative Commons Attribution (CC BY) license (http://creativecommons.org/licenses/by/4.0/). 